\title{
4-Hydroxy-trans-cinnamate Derivatives and Triterpene from Barleria cristata
}

\author{
Nipa Chowdhury ${ }^{1}$, Abdullah Al Hasan ${ }^{2}$, Fakir Shahidullah Tareq ${ }^{3}$, \\ Monira Ahsan ${ }^{1}$ and A.T.M. Zafrul Azam ${ }^{1}$
}

\author{
${ }^{1}$ Phytochemical Research Laboratory, Department of Pharmaceutical Chemistry, Faculty of Pharmacy \\ University of Dhaka, Dhaka-1000, Bangladesh \\ ${ }^{2}$ Department of Pharmacy, Southeast University, Banani, Dhaka, Bangladesh \\ ${ }^{3}$ Department of Marine Biotechnology, University of Science and Technology, 113 Gwahangno \\ Yuseong-gu, Daejeon, Republic of Korea
}

\begin{abstract}
Barleria cristata is an important medicinal plant of Bangladesh. Many compounds of diverse biological activities were isolated from different Barleria species, including irridoids, flavonoids and phenylethanoid derivatives in addition to other groups of chemical constituents. This paper presents the chemical investigation of the whole plants of B. cristata. Classic phytochemical investigation of organic extracts of the whole plants of B. cristata together with spectroscopic methods led to the isolation and characterization of 4-hydroxy-trans-cinnamate derivatives (1-3) and a triterpene, namely oleanolic acid (4).
\end{abstract}

Key words: Barleria, Acanthaceae, aromatic compounds, triterpene.

\section{INTRODUCTION}

Barleria is a genus of plants belonging to the family Acanthaceae. The genus Barleria consists of 28 taxa including 26 species and one sub-species. It is the third largest genus in the family Acanthaceae with 300 species. $^{1,2}$ Balkwill and Balkwill (1997) ${ }^{1}$ reported 32 species from India and Karthikeyan et al. $(2009)^{3}$ enumerated 29 species, one sub-species and six varieties.

Barleria cristata is commonly known as Philippine violet. Blue bell Barleria or Crested Philippine violet and is cultivated as an ornamental plant in villages and gardens. B. cristata is a shrub found widely in subtropical Himalaya, Sikkim, Kashi Hills and southern India at a height of 1,350 meter. The plants are 6-10 meter tall, leaves are dark green on upper surface, lower surface pale green, elliptic to

Correspondence to: A.T.M. Zafrul Azam

Tel. +880-1710-880064; Fax: +880-2-8615583

Email: zafrulazam@du.ac.bd

Dhaka Univ. J. Pharm. Sci. 12(2): 143-145, 2013 (December) narrowly ovate $2.5-10 \mathrm{~cm}$ long, flowers are spinymargined, the inner 2-lobes linear, $7 \mathrm{~mm}$ long, entire margins, corolla violet, pink or white, funnel forms 5 - $5.7 \mathrm{~cm}$ long. ${ }^{4}$ The biological investigation of the plant showed anti-inflammatory, anti-anaemic and anti-toothache, ${ }^{5}$ anti-plasmodial and antioxidant properties. ${ }^{6}$ Previous phytochemical studies with the plant led to the isolation and structure elucidation of flavonoids, phenolic compounds, iridoidal ${ }^{7}$ and phenylethanoid glycosides. ${ }^{8}$

The present study has been undertaken to isolate and identify biologically active secondary metabolites and we, herein, report 4-hydroxy-transcinnamate derivatives (1-3) and a triterpene, namely oleanolic acid (4).

\section{MATERIALS AND METHODS}

General experimental procedure. Preparative TLC was conducted over glass plates coated with silica gel $60 \mathrm{PF}_{254}(0.5 \mathrm{~mm}$ thickness, Merck) and compounds were detected with vanillin $/ \mathrm{H}_{2} \mathrm{SO}_{4}$ spray 
reagent. Gel permeation chromatography was performed using Sephadex LH-20. ${ }^{1} \mathrm{H}$ NMR spectra were acquired in $\mathrm{CDCl}_{3}$ ( $\delta$ values were reported in reference to $\mathrm{CHCl}_{3}$ at $7.25 \mathrm{ppm}$ ) on a Bruker Avance $400 \mathrm{MHz}$ Ultrashield NMR spectrometer equipped with broadband and selective $\left({ }^{1} \mathrm{H}\right.$ and $\left.{ }^{13} \mathrm{C}\right)$ inverse probes.

Plant material. Barleria cristata (L.) was collected from Sylhet, Bangladesh in the month of June, 2012 and was identified by the taxonomist of Bangladesh National Herbarium, Dhaka, Bangladesh. A voucher specimen (Accession No. 37542) of the plant has been deposited there for future reference.

Extraction and isolation. The powdered whole plant $(1500 \mathrm{~g})$ of $B$. cristata was soaked at room temperature with $5 \mathrm{~L}$ of methanol for 13 days and filtered through a cotton plug followed by Whatman filter paper no 1 . The extract was then concentrated with a rotary evaporator. An aliquot of crude methanol extract ( $35 \mathrm{~g}$ ) was fractionated by vacuum liquid chromatographic (VLC) technique using silica gel $60 \mathrm{H}$ (petroleum ether, petroleum ether-ethyl acetate and ethyl acetate in increasing order of polarity). This provided 22 VLC fractions. Following TLC screening of fractions of VLC, fractions 08 and 09 were subjected to column chromatography over lipophilic Sephadex (LH-20) and chloroformpetroleum ether combination as mobile phase. This yielded four compounds 1 (at $\mathrm{R}_{\mathrm{f}}=0.60$ in tolueneethyl acetate 99:1) 2, 3 (at $R_{\mathrm{f}}=0.52$ in toluene-ethyl acetate 95:5) and 4 (at $\mathrm{R}_{\mathrm{f}}=0.2307$ in toluene-ethyl acetate 99:1).

\section{RESULTS AND DISCUSSION}

The chemical study of the whole plant of $B$. cristata led to the identification of three aromatic compounds (4-hydroxy-trans-cinnamate derivatives) and a triterpene characterized as oleanolic acid. Although compounds 1-3 have been tentatively identified, the exact number of methylene group could not be ascertained due to lack of MS data.

The ${ }^{1} \mathrm{H}$ NMR spectral data of compound 1 indicated two doublets $(1 \mathrm{H}$ each $)$ at $\delta 6.28(J=16.0$ $\mathrm{Hz})$ and $7.61(J=16.0 \mathrm{~Hz})$ which are evident of the presence of trans-coupled protons $\mathrm{H}-8$ and H-7, respectively of the cinnamoyl moiety. Two doublets $(2 \mathrm{H}$ each) at $\delta 6.83(J=8.4 \mathrm{~Hz})$ and $7.41(J=8.4 \mathrm{~Hz})$ were assignable to aromatic protons $\mathrm{H}-3$ \& 5 and $\mathrm{H}-2$ $\& 6$, respectively. The above spectral features are in close agreement to those observed for 4-hydroxytrans-cinnamate derivative. ${ }^{9}$ On this basis, the identity of 1 was proposed as a 4-hydroxy-transcinnamic acid alkyl ester. But the actual number of methylene protons of the alkyl group couldn't be determined due to lack of sufficient data.
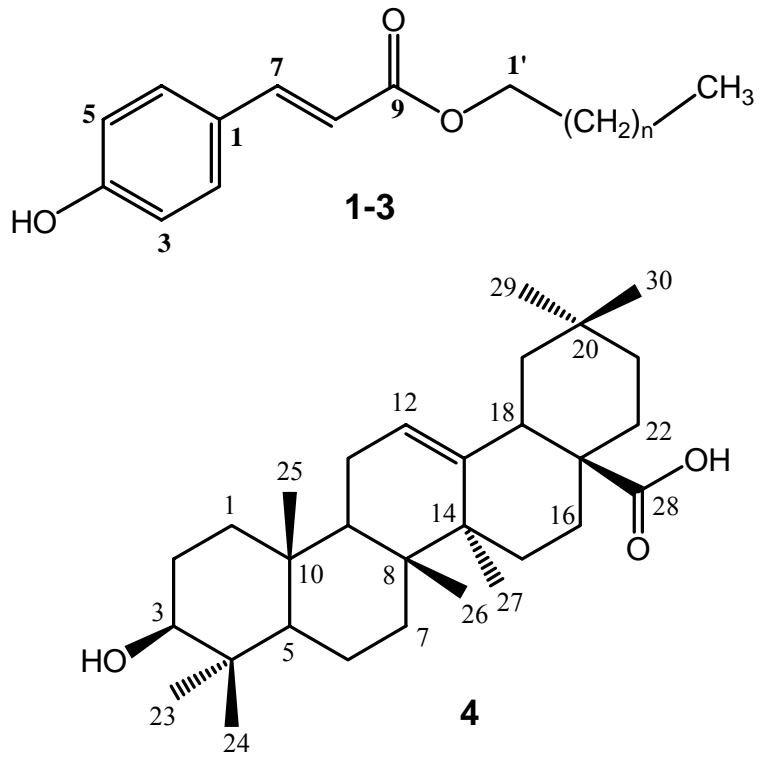

Figure 1. Chemical structure of 4-hydroxy-trans-cinnamate derivatives (1-3) and oleanolic acid (4).

The ${ }^{1} \mathrm{H}$ NMR $\left(\mathrm{CDCl}_{3}, 400 \mathrm{MHz}\right)$ spectrum of sample 2 represent features which indicate it to be a mixer of two para-hydroxy-trans-alkyl cinnamates (here 2 \& 3) closely related to compound 1 . The ${ }^{1} \mathrm{H}$ NMR spectral data (400 MHz, $\mathrm{CDCl}_{3}$ ) of compound $2 \& 3$ indicated two doublets $(1 \mathrm{H}$ each $)$ at $\delta 6.30(\mathrm{~J}=$ $16.0 \mathrm{~Hz})$ and $7.62(J=16.0 \mathrm{~Hz})$ which revealed the presence of trans-coupled protons $\mathrm{H}-8$ and $\mathrm{H}-7$, respectively of the cinnamoyl moiety. Two doublets (2H each) at $\delta 6.83(J=9.0 \mathrm{~Hz})$ and $7.43(J=9.0 \mathrm{~Hz})$ are assignable to aromatic protons $\mathrm{H}-3 \& 5$ and $\mathrm{H}-2$ $\& 6$, respectively. The above spectral features are in close agreement to those observed for 4-hydroxytrans-cinnamate derivative. ${ }^{9}$ 
In addition, the ${ }^{1} \mathrm{H}$ NMR spectral data (400 $\left.\mathrm{MHz}, \mathrm{CDCl}_{3}\right)$ of compound 3 indicated the two doublets $(1 \mathrm{H}$ each $)$ at $\delta 6.30(J=16.0 \mathrm{~Hz})$ and 7.62 $(J=16.0 \mathrm{~Hz})$ are evident of the presence of another trans-coupled protons H-8 and H-7, respectively of the cinnamoyl moiety. Two doublets $(2 \mathrm{H}$ each $)$ at $\delta$ $6.80(J=9.0 \mathrm{~Hz})$ and $7.65(J=9.0 \mathrm{~Hz})$ are assignable to aromatic protons $\mathrm{H}-3$ \& 5 and $\mathrm{H}-2$ \& 6 , respectively. The above spectral features are in close agreement to those observed for 4-hydroxy-transcinnamate derivative. ${ }^{9}$

On this basis, the identity of this sample was proposed as a mixture of two 4-hydroxy-transcinnamic acid alkyl esters. But the actual number of protons of the alkyl group could not be determined due to lack of sufficient data. This is the first report of 4-hydroxy-trans-cinnamic acid alkyl esters (1-3) from this plant.

The ${ }^{1} \mathrm{H}$ NMR (400 MHz, $\mathrm{CDCl}_{3}$ ) of compound 4 showed seven tertiary methyl groups at $\delta 0.75,0.77$, $0.89,0.90,0.92,0.98$ and 1.12 on an oleanane-type carbon skeleton. A doublet of doublet of one proton intensity at $\delta 2.80$ and a triplet $(1 \mathrm{H})$ at $\delta 5.27$ (vinyl proton) were assigned to $\mathrm{H} \alpha-18$ and $\mathrm{H}-12$, respectively, suggesting an olea-12-ene skeleton. An oxymethine proton at $\delta 3.20(d d, J=11.5$ and $4.5 \mathrm{~Hz})$ demonstrated that the compound has at least one hydroxyl group.

Based on the above data and by comparing its spectroscopic data with that reported in literature, ${ }^{10}$ the compound was characterized as oleanolic acid (4). This is the first report of isolation of oleanolic acid from this plant.

\section{REFERENCES}

1. Balkwill, M.J. and Balkwill, K. 1998. A preliminary analysis of distribution pattern in a large, pantropical genus, Barleria L. (Acanthaceae). J. Biogeography 25, 95-110.

2. Mabberley, D.J. 2008. Mabberley's Plant-Book. A portable dictionary of plants, their classification and uses. Third Edition, Cambridge University Press, Cambridge.

3. Karthikeyan, S., Sanjappa, M. and Moorthy, S. 2009. Acanthaceae. In: Flowering Plants of India - Dicotyledons (Acanthaceae - Avicenniaceae). Botanical Survey of India, Kolkata. Volume I, pp. 1-62.

4. Wagner, W.L., Herbst, D.R. and Shomer, S.H. 1999. Manual of the flowering plants of Hawaii. Revised edition, Bernice. Bishop Museum Special Publications: Bishop Museum Press. Honolulu. pp. 1919-1920.

5. Gambhire, M., Juvekar, A. and Wankhede, S. 2008. Evaluation of anti-inflammatory activity of methanol extract of Barleria cristata leaves by in vivo and in vitro methods. Internet J. Pharmacol. 7, 1-4.

6. Charoenchai, P., Vajrodaya, S., Somprasong, W., Mahidol, C., Ruchirawat, S. and Kittakoop, P. 2010. Part 1: Antiplasmodial, cytotoxic, radical scavenging and antioxidant activities of Thai plants in the family Acanthaceae. Planta. Med. 76, 1940-1943.

7. Hemalatha, K., Hareeka, N. and Sunitha, D. 2012. Chemical constituents isolated from leaves of Barleria cristata Linn. Int. J. Pharma and Biosci. 3, 609-615.

8. Abd El-Mawla, A.M.A., Ahmed, A.S., Ibraheim, Z.Z. and Ernst, L. 2005. Phenylethanoid glycosides from Barleria cristata L. callus cultures. Bull. Pharm. Sci. 28, 199-204.

9. Kuo, Y-H., Lo, J-M. and Chan, Y-F. 2002. Cytotoxic components from the leaves of Schefflera taiwaniana. J. Chin. Chem. Soc. 49, 427-431.

10. Gohari, A.R., Saeidnia, S., Hadjiakhoondi, A., Abdoullahi, M. and Nezafati, M. 2009. Isolation and quantificative analysis of oleanolic acid from Satureja mutica Fisch. \& C.A. Mey. J. Med. Plants 8, 65-69. 